made, but localization of an aneurysm to a particular artery is not to be expected.

Treatment.-With such imperfect means of diagnosis little hope can be held out of curative measures. I.igation of the carotid artery has been contemplated (Hutchinson $^{18}$ ) in one case in which a bruit was heard, but operation was refused. It is difficult to conceive how such a procedure could avail when there is such an adequate collateral circulation as the circle of Willis affords.

242 North Sixty-First Street.

\section{TREATMENT OF SYPHILIS OF THE BRAIN *}

A PRELIMINARY REPORT OF THE USE OF MERCURIC CHLORID INTRADURALLY

GUY O'NEIL IRELAND, M.D.C.M., F.T.M.C. Assistant Physician to Western State Hospital, Fort Steilacoom

$$
\begin{aligned}
& \text { FORT STEILACOOM, WASH. } \\
& \text { C. STUART WILSON, M.D. } \\
& \text { TACOMA, WASH. }
\end{aligned}
$$

The proof that general paralysis of the insane is an active syphilitic disease, ${ }^{1}$ together with the advent of intradural medication, has given a new hope in the treatment of these unfortunates.

The choice of technic in intradural treatment lies between the Swift and Ellis method, ${ }^{2}$ the Ravant method, ${ }^{3}$ and the Byrnes method. ${ }^{4}$

The high cost of salvarsan and the scarcity of this product in our Western market have prevented ts from using the Swift and Ellis method to any great extent. We were attracted by the Byrnes method because of its apparent freedom from danger and its low cost.

The patients here reported, twenty-three in number, have all been diagnosed clinically as having general paralysis, and the clinical diagnosis has been accepted as the basis for this work. The patients have not been selected as to the duration of disease, stage, or physical condition; but rather as they came to this particular service. This series represents, however, mostly the so-called third stage.

The technic of treatment is practically the same as described by Byrnes. ${ }^{4}$ About 40 c.c. of blood are taken, under aseptic conditions, from one of the veins of the forearm, and the serum allowed to separate. To 12 c.c. of clear serum is added 1 c.c. of a sterile solution of mercuric chlorid containing $1 / 50$ grain, and 17 c.c. of sterile normal salt solution prepared from freshly distilled water. This total of 30 c.c. is heated to $56 \mathrm{C}$. in a water bath for thirty minutes. Lumbar puncture is performed and from 15 to 30 c.c. of flitid are withdrawn, and the 30 c.c. of diluted mercurialized serum is slowly injected. We have used a 25 c.c. Lüer syringe.

18. Hutchinson: Tr. Clin. Soc., viii, 127.

* The tables showing in detail the clinical and serologic controls in this series are onitted from this articie because of limited space, but will appear in full in the author's reprints.

1. Noguchi: Jour. Exper. Med., 1913, xvii, 232.
2. Swift, Homer E., and Ellis, Arthit W. M.: The Treatment of Syphilitic Affections of the Central Nervous System with Especial Reference to the Use of Intraspinous Injections, Arch. Int. Med., Sept. 15, 1913, p. 331 .

3. Ravaut: Ann de méd., 1914 , No. 1 , p. 49

4. Byrnes, Charles Metcalfe: The Intradural Administration of Nercurialized Serum in the Treatment of Cerebrospinal Syphilis, TH Journal A. M. A., Dec. 19, 1914, p. 2182.
In those cases in which neosalvarsan was given, thic usual Swift and Ellis. technic was used. The serum has been administered within twenty-four hours of its preparation. The interval between injections has been determined by the condition of the patient.

The Wassermann tests were carried out with original technic in one-tenth quantities.

Antigens: Alcoholic extract of beef heart and this same antigen fortified with cholesterin 0.4 per cent.

Patient's blood serum used in 0.1 c.c. unless otherwise noted.

Patient's spinal fluid used in 0.1 c.c., 0.5 c.c. and 1 c.c.

Hemolytic system used was rabbit antisheep.

Complement was from the pooled serum of guineapigs.

Globulin test made according to Nonne. ${ }^{5}$

Cells counted with Thoma-Zeiss hemocytometer.

Lange's colloidal gold test according to Miller and Levy. ${ }^{6}$

In those spinal fluids which had the slightest tinge of blood no cell count, Nonne's or Lange's was done. There were, however, several tubes which on their arrival at the laboratory had perfectly clear fluid but a very slight blood sediment. No cell counts were made on these, but a globulin and Lange's tests were performed.

The following case records show the clinical and serologic findings. Many of these patients had treatment before coming under our observation but their histories are indefinite and do not include intraspinal injections :

CASE 1 (B).-The patient has delusions of grandeur, hears voices, is violent and has to be restrained. Knee jerks are absent, the pupils are of the Arygll Robertson type, therc are fibrillary tremors, the tongue is tremulous, deflected to right, and there is ataxia of speech and gait.

Treatment: 14 intraspinal injections of mercurialized serum; 1 Swift and Ellis 40 per cent. neosalvarsanizet serum; 14 grains of mercury succinimid intramuscularly. Total mercury: intraspinal, $1 / 3$ grain; total serum, 180 c.c. Results: At present the patient is mentally clear, the neurologic signs being absent except that the pupils are unequal. Wassermann, blood serum and spinal fluid tests were negative; the globulin test doubtful, 40 cells; Lange's test positive.

CASE 2 (C).-The patient has delusions of grandeur, hears voices, sees things, shows extreme fabrication, is excitable, weak and anemic. Weight is 86 pounds. Knee jerks + ; pupils, Argyll Robertson. There are fibrillary tremors and ataxic speech and gait.

Treatment: 6 intraspinal injections of mercurialized serum; Swift and Ellis 40 per cent. neosalvarsanized serum; 5 grains of mercury succinimid intramuscularly. Total mercury: intraspinal $1 / 8$ grain; total serum, 120 c.c. Results: The patient is mentally clear, neurologic signs absent except delayed knee jerks. Weight is 119 pounds. Wassermann on blood, 0.2 c.c., positive; spinal fluid, 1 c.c., positive; globulin negative, 10 cells; Lange's positive.

CASE 3 (A).-The patient has delusions of grandeur, is excitable, talkative and filthy. Knee jerks + , pupils normal, reaction + . Ataxic speech and gait. Blood pressure 182 $\mathrm{mm}$. Hg.

Treatment: 4 intraspinal injections of mercurialized serum; 3 Swift and Ellis 40 per cent. neosalvarsanized serum. Total mercury: intraspinal, 1/42 grain, total serum, 84 c.c. Results: clinical and mental improvements, some lessening of neurologic signs. Sudcen death occurred, following cerebral apoplexy.

5. Nonne: Syphilis and the Nervous System, Philadelphia, J. B. Lipinincott Company, Ed. 2

6. Miller and Levy: Bull. Johns Hopkins Hosp., 1914, xx-v, No. 279, p. 133. 
CASE $4(Q)$. - The patient has delusions of grandeur and persecution, hears voices and see things. Knee jerk + , pupils normal, Romberg + . Fibrillary tremors and ataxia are present.

Treatment: 2 intraspinal injections of mercurialized serum, 4 Swift and Ellis 40 per cent. neosalvarsanized serum. Total mercury: intraspina1, 1/25 grain; total serum, 72 c.c. Results : none permanent mentally or physically; Wassermann on blood always negative, spinal fluid positive; globulin positive, 60 cells; Lange's positive; patient went on to usual death of paresis.

CASE $5(\mathrm{~K})$.- The patient has delusions of grandeur and persecution, and is depressed. Knee jerks +. There are fibrillary tremors and ataxia. Blood pressure $173 \mathrm{~mm}$.

Treatment: 8 intraspinal injections of mercurialized serum, 8 grains mercury succinimid intramuscularly. Total mercury: intraspinal, $1 / 6$ grain; total serum, 96 c.c. Results: no particular change. Sudden death following cerebral apoplexy.

CASE $6(Y)$.-The patient has delusions of grandeur and is restless. Knee jerks +, pupils, Argyll Robertson; Romberg + There are fibrillary tremors and ataxic speech and gait.

Treatment: 5 intraspinal injections of mercurialized serum, 5 grains of mercury succinimid intramuscularly. Total mercury; intraspinal, 1/10 grain; total serum, 60 c.c. Results: Mental improvement except that the patient is emotional; neurologic signs are absent, Wassermann on blood is doubtful; spinal fluid positive; globulin partially positive, 40 cells; Lange's positive.

CASE 7 (R).-The patient has delusions of grandeur and is restless. Knee jerks +, pupils Argyll Robertson, Romberg + There are tremors and ataxia.

Treatment: 6 intraspinal injections of mercurialized serum; 6 grains mercury succinimid intramuscularly. Total mercury; intraspinal, $1 / 8$ grain; total serum, 72 c.c. Results: no change clinical or serologic.

CASE 8 (G).-The patient has delusions of grandeur and persecution, high sexual desire and is depressed. Knee jerks + , pupils Aryg1l Robertson, Romberg + . Ataxia and tremors are present.

Treatment: 9 intraspinal injections of mercurialized serum; 9 grains of mercury succinimid intramuscularly. Total mercury: intraspinal, $\%$ grain; total serum, 108 c.c. Results At times patient is rational, delusions less active. Knee jerks +. Argyll Robertson +, Romberg -. Ataxia is not present; Wassermann on the blood has always been negative, on the spinal fluid, variable; globulin faintly positive, 50 cells; Lange's variable.

CASE 9 (T).-The patient has no delusions but is depressed. Knee jerks +, pupils, Argyll Robertson, Romberg +. There are fibrillary tremors and ataxia.

Treatment: 7 intraspinal injections of mercurialized serum; 7 grains mercury succinimid intramuscularly. Total mercury: intraspinal $1 / 7$ grain; total serum, 84 c.c. Results: Patient is mentally improved. Knee jerks -, Romberg pupils Argyll Robertson; tremors and ataxia less. Wassermann on blood partially positive; spinal fluid positive; globulin positive, 90 cells; Lange's positive.

CASE $10(\mathrm{~F})$.- The patient has delusions of grandeur and persecution, sees and hears things and is violent. Knee jerks +, pupils, Argyll Robertson, reaction + . There are tremors and ataxia.

Treatment: 13 intraspinal injections of mercurialized serum; 13 grains of mercury succinimid intramuscularly. Total mercury: intraspinal, $1 / 4$ grain; total serum, 156 c.c. Results: No change clinically, Wassermann on blood partially positive; spinal fluid positive; globulin partially positive, 20 cells; Lange's positive.

CASE 11 (O).-The patient has delusions of grandeur, sees and hears things, is violent and has to be restrained. Knee jerks are retarded, the pupils are normal. Ataxia and tremors are present.

Treatment: 4 intraspinal injections of mercurialized scrum; 3 grains mercury succinimid intramuscularly. Total mercury: intraspinal, 1/12 grain; total serum, 48 c.c. Results: No delusions, no hallucinations, clean, bright, neurologic signs, ataxia and tremor less, otherwise no change. Wassermann on blood partially positive; spinal fluid positive; globulin positive, 11 cells; Lange's positive.

CASE 12 (M).-The patient has delusions of grandeur, sees and hears things and varies from extreme emotion to elation. Knee jerks +, pupils, Argyll Robertson, Romberg +. Ataxia and tremors are present.

Treatment: 5 intraspinal injections of mercurialized serum; 5 grains mercury succinimid intramuscularly. Total mercury: intraspinal, $1 / 10$ grain; total serum, 60 c.c. Results: slight mental improvement, neurologic; Argyll Robertson Romberg - . Ataxia and tremors gone; Wassermann always negative on blood and spinal fluid; globulin positive, 98 cells; Lange's positive.

CASE $13(\mathrm{P})$.- The patient has delusions of grandeur and persecution and is violent. Knee jerks + , pupils, Argyll Robertson. Tremors are present.

Treatment: 13 intraspinal injections of mercurialized serum; 2 Swift and Ellis neosalvarsanized serum; 13 grains mercury succinimid intramuscularly. Total mercury: intraspinal. $1 / 4$ grain; total serum, 180 c.c. Results: no change mentally or neurologically; Wassermann on blood, doubtful; spinal fluid positive; globulin doubtful, 160 cells; Lange's positive.

CASE 14 (W)-The patient has delusions of grandeur, is dull and confused. Knee jerks + , Romberg +, pupils, Argyll Robertson; there are ataxia and tremors.

Treatment: 5 intraspinal injections of mercurialized serum; 5 grains mercury succinimid intramuscularly. Total mercury: intraspinal, 1/10 grain; total serum, 60 c.c. Results: no change mentally, or physically; Wassermann on blood and spinal fluid positive; globulin positive, 30 cells; Lange's positive.

CASE 15 (S).-The patient has delusions of grandeur and is violent. Knee jerks --, Romberg + ; pupils, Argyll Robertson. There are ataxia and tremors.

Treatment : 7 intraspinal injections of mercurialized serum; 5 grains mercury succinimid intramuscularly. Total mercury : intraspinal, $1 / 4$ grain; total serum, 84 c.c. Results: Mentally patient is somewhat improved; knee jerks normal; Romberg -, Argyll Robertson, retarded. There is no ataxia or tremor; Wassermann on blood doubtfully positive, spinal fluid positive; globulin positive, 190 cells; Lange's positive.

CASE 16 (U).-The patient has delusions of grandeur, is depressed and untidy. Knee jerks -, Romberg + . The pupils are normal, there is ataxia and tremor.

Treatment : 4 intraspinal injections of mercurialized serum; 4 grains mercury succinimid intramuscularly. Total mercury: intraspinal, 1/22 grain; total serum, 48 c.c. Results: Patient is mentally improved, ataxia and tremor gone; knee jerks normal, Romberg -; pupil, Argyll Robertson. Wassermann on blood and spinal fluid positive; globulin positive, 90 cells; Lange's positive.

CASE $17(\mathrm{H})$.- The patient has delusions of grandeur and is depressed. Knee jerks -, pupils, Argyll Robertson; Romberg + . There are ataxia and tremors.

Treatment: 12 intraspinal injections of mercurialized serum; 11 grains mercury succinimid intramuscularly. Total mercury: intraspinal, $1 / 4$ grain; total serum, 144 c.c. Results: Delusions are not so pronounced, patient is more tidy, ataxia is less. Wassermann on blood is doubtfully positive; on spinal fluid positive; globulin positive, 70 cells; Lange's negative.

CASE 18 (V).-The patient has delusions of grandeur, hears things, and is untidy. Knee jerks +, pupils normal, Romberg + . There are ataxia and tremors.

Treatment: 6 intraspinal injections of mercurialized serum; 6 grains mercury succinimid. Total mercury: intraspinal, 1/8 grain; total serum, 72 c.c. Results: Patient is mentally clear, knee jerks - Romberg -; pupils normal; no ataxia or tremor; Wassermann on blood and spinal fluid positive; globulin positive, 10 cells; Lange's positive. 
CASE 19 (E).-The patient has delusions of grandeur, is violent and untidy. Knee jerks + , pupils, Argyll Robertson, Romberg + . There are ataxia and tremors.

Treatment: 16 intraspinal injections of mercurialized serum; 1 Swift and Ellis 40 per cent. neosalvarsanized serum, 16 grains mercury succinimid intramuscularly. Total mercury : intraspinal, $1 / 3$ grain; total serum 204 c.c. Results : The patient is mentally clear, pupils normal; knee jerks + , Romberg + . There is some tremor: Wassermann on hlood always negative, spinal fluid partially positive; globulin partially positive, 20 cells; Lange's positive.

CASE 20 (L).-The patient has delusions of grandeur and is violent. Knee jerks +, pupils, Argyll Robertson, Romberg t. Ataxia and tremors are present.

Treatment: 16 intraspinal injections of mercurialized serum; 16 grains mercury succinimid intramuscularly. Total mercury: intraspinal, $1 / 3$ grain ; total serum, 192 c.c. Results: Variable improvement mentally, ataxia and tremors gone. Knee jerks, pupils, Argyll Robertson, Romberg +. Wassermann on blood partially positive; spinal fuid positive; globulin positive. 60 cells; Lange's positive.

CASE 21 (X).-The patient has delusions of grandeur and is depressed. Knee jerks +, pupils Argyll Robertson, Romberg + . There are ataxia and tremors.

Treatment : 5 intraspinal injections of mercurialized serum; 5 grains mercury succinimid intramuscularly. Total mercury: intraspinal, 1/10 grain; total serum, 60 c.c. Results: Patient is brighter, delusions are indefinite; no change in physical signs. Wassermann on blood, partially positive; spinal fluid positive; globulin positive, 40 cells; Lange's positive.

CASE 22 (D).-The patient has delusions of grandeur, is restless and hears things. Knee jerks +; pupils, Argyll Robertson; Romberg + . There are ataxia and tremors.

Treatment: 13 intraspinal injections of mercurialized serum; 13 grains mercury succinimid intramuscularly. Total mercury: intraspinal, $1 / 4$ grain; total serum, 152 c.c. Results: no change, Wassermann in blood always negative, spinal fluid variable; globulin positive, 20 cells; Lange's positive.

CASE 23 (I).-The patient has no delusions, but is unconscious at times and has flighty and disconnected speech. Knee jerks +; pupils, Argyll Robertson, Romberg +. There are fibrillary tremors but no ataxia.

Treatment: 2 intraspinal injections of mercurialized serum; 7 Swift and Ellis 40 per cent. neosalvarsanized serum; 11 neosalvarsan intravenously. Total mercury: intraspinal, 1/y grain; total serum, 108 c.c. Results: Patient is normal mentally; knee jerks slightly exaggerated, and pupils still sluggish, Wassermann on blood negative; spinal fluid positive in 1 c.c.; globulin negative, 30 cells; Lange's negative.

Practically all mercurialized serum given prior to March 30 with the exception of that given to Patient 1 was prepared from pooled blood serum. The reactions were those of severe pain in back, legs and testes. About March 21 all patients receiving treatment had very marked depression and constipation. This depression was so severe that a two weeks' interval was necessary before resuming treatment. The spinal fluid of one of the donors at that time was orange yellow, but did not give hemoglobin reaction. Subsequently his blood was not included in the pool and no recurrence of a universal depression followed. When we changed to autogenous serum most of the patients remarked on the lessened reaction.

In all but three cases there has been a clinical improvement some time during their treatment. This improvement has taken place by the fifth treatment in 75 per cent. of the remaining twenty cases. There has been a tendency to a return to their former clinical state some time later in 40 per cent. of those improved.

In 15 per cent. the improvement reached a certain point and seemed to proceed no further as in Patient
D., who improved after his sixth treatment but has remained stationary through his following seven treatments.

The serologic improvement has not been so marked or so constant as the clinical. Of the fifteen patients who received the $1 / 30$ grain mercuric chlorid intradurally, 46 per cent. had a severe reaction and 54 per cent. 110 more severe than usual; 61 per cent. had their Wassermann in the spinal fluid temporarily converted to a negative, while 38 per cent. were not immediately influenced. We did not continue the $1 / 30$ grain injections because the reactions were too severe.

The marked fluctuations in the pupillary reactions and knee jerks are of interest, as an exaggerated knee jerk may become temporarily lost and vice versa. We have not noticed any constant relation between the globulin reaction, cells and colloidal gold test

In only two cases out of the twenty-three have we seemed to be able to convert the colloidal gold test to a more or less permanent negative. The globulin reaction and plencytosis were very variable and seemed to follow no definite course.

\section{CONCLUSIONS}

The injections of $1 / 50$ grain of mercuric chlorid intradurally, by the method of Byrnes; is apparently safe.

Autogenous serum is much preferable to pooled serum.

The reactions are a little more severe than following the Swift and Ellis method of 40 per cent. salvarsanized serum.

A clinical improvement usually appears after about five treatments; 75 per cent. of our cases showed this improvement.

A tendency to relapse to their former condition was observed in about 40 per cent. of those at first improved.

The clinical improvement is more marked and rapid than the serologic.

How permanent the improvement will be we are unable to state, as the time of observation is too short.

We are encouraged to continue this study, however, and a later report will be given on these patients after further treatment and observation.

The colloidal gold test is the most resistant to change.

It is possible to have clinical general paralysis with a negative Wassermann reaction in blood serum and spinal fluid, but in this series the colloidal gold test was uniformly positive.

Honor to Spanish Physician.-The Siglo Medico of July 31 relates that the town physician of Cercedilla, Spain, D:. A. Cañadas, was treating a pauper child of 3 for a severe burn; deeming a skin graft necessary, he took it from his own person and cured the child. The local authorities and the people of Cercedilla published an appeal in a Madrid paper petitioning for an official reward for this act of altruism. The minister of the interior, the governor and municipality of Madrid and others, including the medical profession, organized a banquet in his honor at the Escorial. One of the lay speakers said that Cañadas' act "should be engraved with letters of gold in the pages of humanity." Cañadas illustrated the technic with which he was able to cut from his own skin the extensive piece required. Steps are being taken to have the cross of the order of Beneficienc a conferred on him. 